\title{
Critical Behavior of the Thermoelectric Transport Properties in Amorphous Systems near the Metal-Insulator Transition
}

\author{
C. Villagonzalo ${ }^{1}{ }^{\star}$, R. A. Römer ${ }^{1}$, M. Schreiber ${ }^{1}$, A. MacKinnon ${ }^{2}$ \\ 1 Institut für Physik, Technische Universität, 09107 Chemnitz, Germany \\ 2 Blackett Laboratory, Imperial College, Prince Consort Rd., London SW7 2BZ, U.K.
}

\begin{abstract}
The scaling behavior of the thermoelectric transport properties in disordered systems is studied in the energy region near the metal-insulator transition. Using an energydependent conductivity $\sigma$ obtained experimentally, we extend our linear-response-based transport calculations in the three-dimensional Anderson model of localization. Taking a dynamical scaling exponent $z$ in agreement with predictions from scaling theories, we show that the temperature-dependent $\sigma$, the thermoelectric power $S$, the thermal conductivity $K$ and the Lorenz number $L_{0}$ obey scaling.
\end{abstract}

The scaling description [1] of disordered systems, e.g. the Anderson model of localization, has cultivated our understanding of transport properties in such systems [2, 3]. According to the scaling hypothesis, the behavior of the d.c. conductivity $\sigma$ near the metal-insulator transition (MIT) in the Anderson model can be described by only a single scaling variable. As a result of the scaling theory, the dynamical conductivity in the three-dimensional (3D) Anderson model behaves as [4, 5]

$\frac{\sigma(t, T)}{T^{1 / z}}=\mathcal{F}\left(\frac{t}{T^{1 / \nu z}}\right)$

where $T$ is the temperature and $t$ is the dimensionless distance from the critical point. For example, $t=$ $\left|1-E_{F} / E_{c}\right|$ where $E_{F}$ and $E_{c}$ are the Fermi energy and the mobility edge, respectively. The parameter $\nu$ is the correlation length exponent, which in $3 \mathrm{D}$ is equivalent to the conductivity exponent, $\sigma \propto t^{\nu}$, and $z$ is the dynamical exponent, $\sigma \propto T^{1 / z}$. It was further demonstrated that not only $\sigma(t, T)$ obeys scaling in the 3D Anderson model but also the thermoelectric power $S(t, T)$ [6, [], the thermal conductivity $K(t, T)$ and the Lorenz number $L_{0}(t, T)[7]$. However, despite the quality of the scaling of $\sigma$, we obtained an unphysical value for $z$ [7]. Scaling arguments for noninteracting systems predict $z=d$ in $d$ dimensions [4,5]. But we found [7] $z=1 / \nu \ll 3$. In addition, values of $S(T)$ [8, 9] are at least an order of magnitude larger than in measurements of doped semiconductors 10] and amorphous alloys 11, 12.

In what follows, we show that we obtain the right order of magnitude [13] and good scaling for these thermoelectric transport properties by using a "modified" critical behavior of $\sigma$ in the linear-response formulation for the Anderson model based on experimental data.

* Permanent address: National Institute of Physics, University of the Philippines, Diliman, 1101 Q. C., Philippines
In the linear-response formulation, the thermoelectric transport properties can be determined from the kinetic coefficients $L_{i j}$ [9], i.e.,

$$
\begin{array}{ll}
\sigma=L_{11}, & K=\frac{L_{22} L_{11}-L_{21} L_{12}}{e^{2} T L_{11}}, \\
S=\frac{L_{12}}{|e| T L_{11}}, & \text { and } \quad L_{0}=\frac{L_{22} L_{11}-L_{21} L_{12}}{\left(k_{B} T L_{11}\right)^{2}} .
\end{array}
$$

The $L_{i j}$ relate the induced charge and heat current densities to their sources such as a temperature gradient 9]. In the absence of interactions and inelastic scattering processes, the $L_{i j}$ are expressed as [14, 15, 16]

$$
\begin{array}{r}
L_{i j}=(-1)^{i+j} \int_{-\infty}^{\infty} A(E)[E-\mu(T)]^{i+j-2} \\
\cdot\left[-\frac{\partial f(E, \mu, T)}{\partial E}\right] d E,
\end{array}
$$

for $i, j=1,2$, where $\mu$ is the chemical potential of the system, $f(E, \mu, T)$ is the Fermi distribution function, and $A(E)$ describes the system dependent features. In the Anderson model, one sets $A(E)$ to be equal to the critical behavior of $\sigma(E) \propto\left|1-E / E_{c}\right|^{\nu}$ [2]. Note, however, that this behavior near the MIT does not contain a $T$ dependence. Hence, the $T$ dependence of the $L_{i j}$ in Eq. (3) is merely due to the broadening of $f$ and the $T$ dependence of $\mu$. The latter stems from the structure of the density of states, variations in which yield only negligible changes in $L_{i j}$ [13]. Thus, in order to model a correct $T$ dependence of the $L_{i j}$ and, consequently, the transport properties, we need to reconsider what $A(E)$ should be.

A suitable $\sigma(E)$ may be obtained from appropriate experimental data. The recent measurements of $\sigma$ by Waffenschmidt et al. 17] in Si:P at the MIT under uniaxial stress show that $\sigma(t, T)$ obeys scaling with $\nu=1 \pm 0.1$ and $z=2.94 \pm 0.3$. Obtaining (i) $z \approx d=3$ in good agreement with scaling arguments [4,5] and (ii) $\nu$ which also agrees reasonably well with the numerical results for noninteracting systems $18,19,20$ makes the experimental data in Ref. [17] an excellent empirical model for $A(E)$. In those experiments, $t$ in $\sigma(t, T)$ is given in terms of the uniaxial stress and its critical value near the MIT. Here, we derive a functional form of $\sigma(E)$ by constructing a polynomial fit or a spline curve of the experimental data and setting $t=\left|1-E / E_{c}\right|$. When using this data 


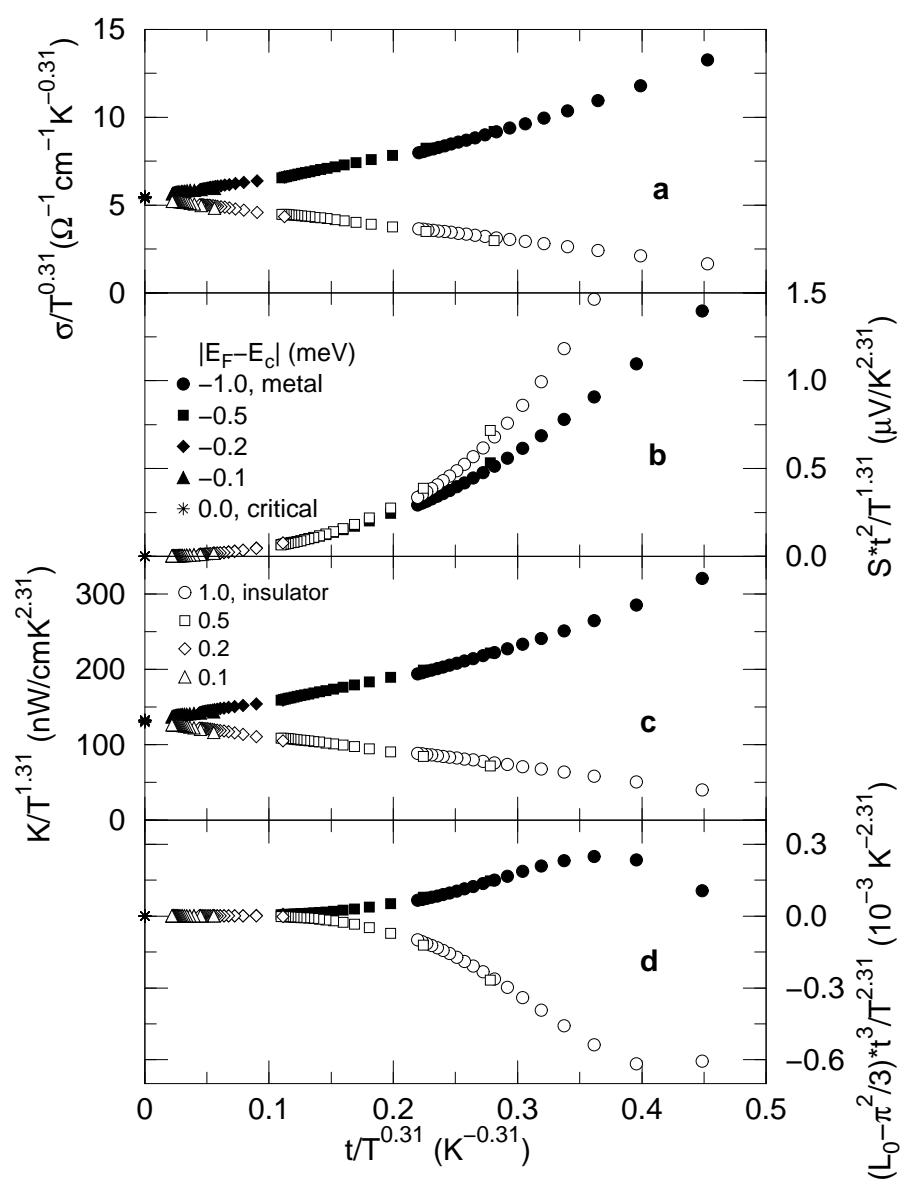

Fig. 1 Scaling of thermoelectric transport properties where $t=\left|1-E_{F} / E_{c}\right|$. The different symbols denote the relative positions of various values of the Fermi energy $E_{F}$ with respect to the mobility edge $E_{c}$.

as input for Eq. (3) the difference in the order of magnitude in $S$ as compared to experiments is removed [13. Thus, following the approach of Ref. [13], we now study whether the $T$ dependence of $\sigma(E, T)$ can be scaled as in Eq. (11) and whether $S, K$ and $L_{0}$ also obey scaling.

In Fig. 1 we show that $\sigma, S, K$ and $L_{0}$ data for different $t$ and $T$ parameters collapse onto scaling curves when plotted as a function of $\left|1-E_{F} / E_{c}\right| / T^{0.31}$. For each figure, we clearly obtain two branches, one for the metallic regime and another for the insulating regime. As depicted in Fig. 11a, $\sigma$ satisfies Eq. (1). With $\nu=1$ in accordance with the experiment in Ref. [17], $1 / \nu z=0.31$ gives $z=3.2$. This is in good agreement with the prediction $z=3$ for 3D noninteracting systems. Furthermore, it indicates that the Harris criterion [21], $\nu z>1$, is satisfied which in turn implies a sharp MIT.

The prefactor in $K$ in Fig. 1 c verifies that $\sigma$ and $K / T$ behave similarly as the MIT is approached. This confirms earlier results in the 3D Anderson model from various methods [9,22]. Meanwhile, the prefactors in both $S$ and $L_{0}$ in Figs. 1 $\mathrm{b}$ and 11d have not been observed in the respective scaling curves in the $3 \mathrm{D}$ Anderson model [7]. The prefactors serve as corrections to the scaling of
$S$ and $L_{0}$ when an appropriate $\sigma(E, T)$ is used as input in Eq. (3).

As shown in Fig. 11d in accordance also with the results in Ref. 13], $L_{0} \rightarrow \pi^{2} / 3$, as the MIT is approached from the metallic or the insulating regime. This is the expected value in the Sommerfeld free electron theory. It is different from the result for the unmodified 3D Anderson model [9] for which the magnitude of $L_{0}$ depends on $\nu$ [9].

In conclusion, we find that by modifying $\sigma(E, T)$ in our calculations, the thermoelectric transport properties near the MIT obey scaling.

Acknowledgements The authors are grateful for the support of the DFG through Sonderforschungsbereich 393, the DAAD, the British Council and the SMWK.

\section{References}

1. E. Abrahams, P. W. Anderson, D. C. Licciardello, and T. V. Ramakrishnan, Phys. Rev. Lett. 42, (1979) 673.

2. B. Kramer and A. MacKinnon, Rep. Prog. Phys. 56, (1993) 1469.

3. P. A. Lee and T. V. Ramakrishnan, Rev. Mod. Phys. 57, (1985) 287.

4. F. Wegner, Z. Phys. B 25, (1976) 327.

5. D. Belitz and T. R. Kirkpatrick, Rev. Mod. Phys. 66, (1994) 261.

6. U. Sivan and Y. Imry, Phys. Rev. B 33, 551 (1986).

7. C. Villagonzalo, R. A. Römer, and M. Schreiber, Ann. Phys. (Leipzig) 8, (1999) SI-269.

8. J. E. Enderby and A. C. Barnes, Phys. Rev. B 49, (1994) 5062.

9. C. Villagonzalo, R. A. Römer, and M. Schreiber, Eur. Phys. J. B 12, (1999) 179.

10. M. Lakner and H. v. Löhneysen, Phys. Rev. Lett. 70, (1993) 3475.

11. C. Lauinger and F. Baumann, J. Phys.: Condens. Matter 7, (1995) 1305.

12. G. Sherwood, M. A. Howson, and G. J. Morgan, J. Phys.: Condens. Matter 3, (1991) 9395.

13. C. Villagonzalo, R. A. Römer, M. Schreiber and A. MacKinnon, preprint (2000), cond-mat/0006083.

14. G. V. Chester and A. Thellung, Proc. Phys. Soc. 77, (1961) 1005.

15. R. Kubo, J. Phys. Soc. Japan 12, (1957) 570.

16. D. A. Greenwood, Proc. Phys. Soc. 71, (1958) 585.

17. S. Waffenschmidt, C. Pfleiderer, and H. v. Löhneysen, Phys. Rev. Lett. 83, (1999) 3005.

18. K. Slevin and T. Ohtsuki, Phys. Rev. Lett. 82, (1999) 382; 82, (1999) 669.

19. F. Milde, R. A. Römer, and M. Schreiber, Phys. Rev. B 61, (2000) 6028.

20. F. Milde, R. A. Römer, M. Schreiber, and V. Uski, Eur. Phys. J. B, (2000), 685.

21. A. B. Harris, J. Phys. C 7, (1974), 1671.

22. G. Strinati and C. Castellani, Phys. Rev. B 36, (1987) 2270 . 